\title{
Multivalent Directed Assembly of Colloidal Particles**
}

\author{
Hirotaka Ejima, Joseph J. Richardson and Frank Caruso*
}

\section{Keywords:}

Colloids · Nanoparticles - Nanostructures ·

Noncovalent interactions · Self-assembly

Nature's building blocks ubiquitously self-assemble into diverse structures in the world around us. In naturally occurring assemblies, directional interactions play a pivotal role in guiding structural complexity and functional specificity at various length scales. Yet, it is still a challenging task in materials science to create building blocks that predictably assemble into desired highly programmable suprastructures. Herein, we highlight an inventive concept for controlling the directional assembly of multivalent colloidal particles, which has recently been reported by Pine and coworkers. ${ }^{[1]}$

Micro- and nano-scale particles, made from organic, inorganic, or hybrid materials, represent an important class of building blocks for a range of applications, including optoelectronics, catalysis, biosensing and drug delivery. ${ }^{[2]}$ Well-defined integration of these materials into complex assemblies is a key step toward the realization of advanced functional materials. Recent progress in the synthesis of monodisperse colloidal particles with programmable sizes, shapes and compositions has paved the way for thermodynamically driven suprastructure assemblies with predictable morphologies.

The directional assembly of colloidal building blocks has been realized through the utilization of either anisotropic shapes (e.g., buckling, ${ }^{[3]}$ faceting ${ }^{[4]}$ ) or specific interactions (e.g., surface roughness, ${ }^{[5]}$ surface patterning $\left.{ }^{[6]}\right)$. The dimension dependent physical and chemical properties of the particles, along with the reaction conditions, govern how these particles self-assemble, and the structures they form. Like artificial atoms, the controllable anisotropy of the particles allows for simulating reaction kinetics. ${ }^{[7]}$

Although diverse structures have been formed from different classes of self-assembling particles, no single class of particles has afforded the three-dimensional (3D) directional bonding that mimics the comprehensive range of atomic bonds. This was recently overcome by Wang and co-workers through the creation of patchy particles with 'valency', that is, a controllable number of reactive sites per particle. ${ }^{[1]}$

[*] Dr. Hirotaka Ejima, Joseph J. Richardson, Prof. Frank Caruso*

Department of Chemical and Biomolecular Engineering The University of Melbourne, Victoria, 3010 (Australia) Fax: (+61) 3-8344-4153

E-mail: fcaruso@unimelb.edu.au

[**] F.C. acknowledges funding from the Australian Research Council under the Australian Laureate Fellowship scheme, and H.E. acknowledges support from the Japan Society for Promotional Science for a Postdoctoral Fellowship.
It was previously shown that polystyrene particles reproducibly self-assemble into geometric shapes by an emulsion-evaporation method, ${ }^{[8]}$ shown in Figure 1a. A discrete number of polystyrene particles $(n)$ were trapped inside toluene emulsions in water. To minimize the second moment of mass distribution as toluene evaporates, the particles undergo dense packing into identical polyhedrons containing $n$ particles. In a novel approach, Wang et al. ${ }^{[1]}$ converted these clusters into atomistic building blocks with reactive patches (Figure 1b) using a two-stage swelling and polymerization process. ${ }^{[9]}$ These patchy clusters required further separation and isolation based on $n$ by density gradient centrifugation. The amount of styrene monomer introduced dictates the size of the non-reactive polystyrene surface, and correspondingly the size of the reactive polystyrene patches. Patches were specifically functionalized with DNA containing singlestranded "sticky" ends, thereby creating 'valency'. ${ }^{[1]}$

The geometries of these patchy particles resemble the arrangements of bonds around atoms. Monovalent $s$ and multivalent $s p, s p^{2}, s p^{3}, s p^{3} d, s p^{3} d^{2}, s p^{3} d^{3}$ hybridized orbitals were replicated by the assembled clusters adopting parallel symmetries (Figure 1b). Mixing particles with complementary sticky ends lead to analogues of molecules such as AB-type (e.g., $\mathrm{HCl}), \mathrm{AB}_{2}$-type $\left(\mathrm{CO}_{2}\right), \mathrm{AB}_{3}$-type $\left(\mathrm{SO}_{3}\right)$ and $\mathrm{AB}_{4}$-type $\left(\mathrm{CCl}_{4}\right)$. Furthermore, colloidal analogues of alternating copolymers were obtained from complementary divalent particles (Figure 1c). Divalent particles with a large patch size could also accommodate two monovalent particles per patch, forming ethylene-like structures.

The kinetics of $\mathrm{AB}_{3}, \mathrm{AB}_{4}$ and copolymer formation were investigated via real-time monitoring with optical microscopy. Since the hybridization of complementary DNA strands $\left(T_{\mathrm{m}}=50{ }^{\circ} \mathrm{C}\right)$ is the driving force for bonding, the assembly was monitored at $25^{\circ} \mathrm{C}$ immediately after rapid quenching from dissociation at $55^{\circ} \mathrm{C}$. The estimated reaction times for $\mathrm{AB}_{4}$ colloidal molecule formation from the Smoluchowski equation are $3.4 \mathrm{~min}, 6.5 \mathrm{~min}$, and $16 \mathrm{~min}$ for the first, second and third monovalent particles to attach, respectively, which is consistent with the real-time monitoring experiments.

As noted by Wang et al., improving the yield and separation methods for the patchy particles is of practical importance. They also suggest that colloids with different characteristics including size, color, chemical functionality and electrical conductivity could be further linked to the patchy building blocks, as long as they can be functionalized with DNA. ${ }^{[1]}$ These patchy particles can be disassembled by heat, but future patchy particles could act as storage devices with electromagnetically controlled (dis)assembly, 


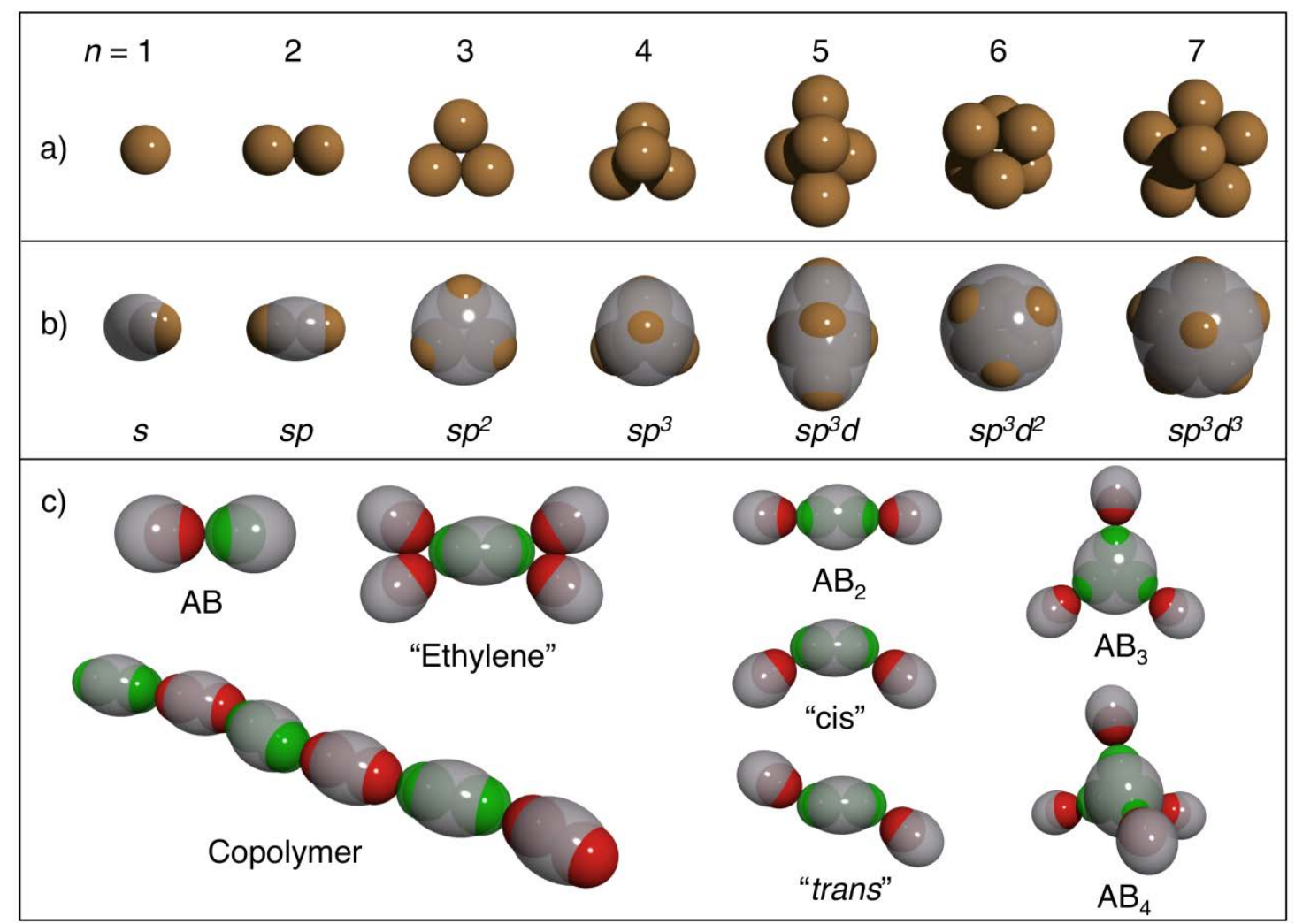

Figure 1. Schematic representation of the directional assembly of colloidal particles synthesized by Wang et al. ${ }^{[1]}$ a) The assembled clusters from $n$ particles. $n=1$, singlet, $n=2$, doublet, $n=3$, triangle, $n=4$, tetrahedron, $n=5$, triangular dipyramid, $n=6$, octahedron; $n=7$, pentagonal dipyramid. b) The colloidal building blocks with the inherent symmetries of their parent clusters. Corresponding hybridized orbitals are shown below. c) The assemblies obtained from the building blocks analogous to molecules.

utilizing light or an electric current. Wang et al. envision that assembled structures analogous to tetrahedrally coordinated diamond crystals with a full 3D photonic band-gap, could be formed by using patchy particles $(n=4)$ with self-complementary DNA (palindrome strands). ${ }^{[1]}$ Similarly, crystals with programmed distributions of 3D defects could be of interest for photonics, catalysis, or electronics, and could be built using metallic particles rather than polystyrene. To date, the methodology for creating patchy particles used by Wang et al. requires synthesis by radical polymerization, ${ }^{[1]}$ but it is expected that this methodology will be expanded to incorporate diverse materials in the future.

To extend the research toward more advanced molecular models, particle affinities paralleling electronegativity could be engineered by varying the quantity of mismatched base pairs in between complementary DNA strands. Other non-covalent and covalent interactions such as hydrophobic, electrostatic, hydrogen bonding, antigen-antibody, sugar-lectin, thiol/disulfide and alkyne-azide coupling should be candidates for controlling equilibration kinetics, improving complex particle yield, or for tuning specificity and functionality. For example, it would be of interest to incorporate stimuli-responsiveness into the directional bonds in between particles, utilizing $\mathrm{pH}$, temperature, light or ionic strength as stimuli. Anisotropic materials have recently received considerable interest in drug delivery, ${ }^{[10]}$ and these multivalent patchy particles and their assembled clusters might possess distinct characteristics in the biological domain, such as directional cellular uptake or reducing phagocytic association.

The concept of particles with valency, introduced by Wang et al., ${ }^{[1]}$ represents a significant step forward in the field of directed assemblies. This simple, yet novel platform will allow the scientific community to create molecule analogues that are, in many ways, potentially as useful and diverse as molecules themselves.
Received: ((will be filled in by the editorial staff))

Published online on ((will be filled in by the editorial staff))

[1] Y. Wang, Y. Wang, D. R. Breed, V. N. Manoharan, L. Feng, A. D. Hollingsworth, M. Weck, D. J. Pine, Nature 2012, 491, 51-55.

[2] F. Li, D. P. Josephson, A. Stein, Angew. Chem. Int. Ed. 2011, 50, 360388.

[3] S. Sacanna, W. T. M. Irvine, P. M. Chaikin, D. J. Pine, Nature 2010, 464, 575-578.

[4] P. F. Damasceno, M. Engel, S. C. Glotzer, Science 2012, 337, 453-457.

[5] D. J. Kraft, R. Ni, F. Smallenburg, M. Hermes, K. Yoon, D. A. Weitz, A. van Blaaderen, J. Groenewold, M. Dijkstra, W. K. Kegel, Proc. Natl. Acad. Sci. U. S. A. 2012, 109, 10787-10792.

[6] Q. Chen, S. C. Bae, S. Granick, Nature 2011, 469, 381-384.

[7] Q. Chen, J. K. Whitmer, S. Jiang, S. C. Bae, E. Luijten, S. Granick, Science 2011, 331, 199-202.

[8] V. N. Manoharan, M. T. Elsesser, D. J. Pine, Science 2003, 301, 483487.

[9] J. Ugelstad, K. H. Kaggerud, F. K. Hansen, A. Berge, Makromol. Chem. 1979, 10, 215-234.

[10] J. P. Best, Y. Yan, F. Caruso, Adv. Healthcare Mater. 2012, 1, 35-47. 
Hirotaka Ejima, Joseph J. Richardson, Frank Caruso* Page -

Page

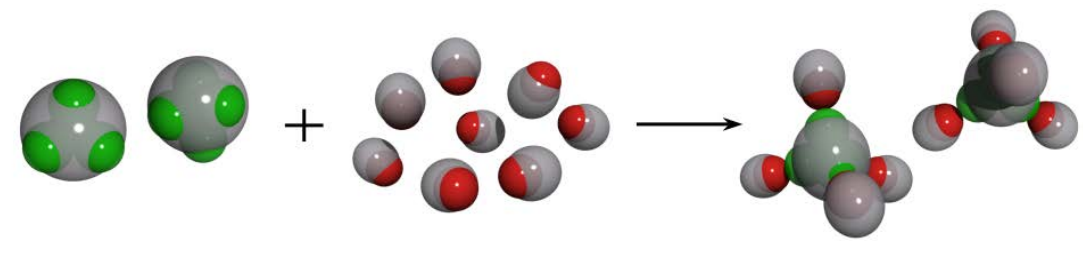

Multivalent Directed Assembly of Colloidal Particles

Artificial molecule analogues: Colloidal particles with a determined number of sticky sites arranged in precise geometries analogous to atoms were created. The kinetics of colloidal reactions related to specific chemical reactions (see pictures) were investigated using optical microscopy. This general platform technology should allow for a broad range of scientifically and practically useful 3D colloidal assemblies to be synthesized and investigated. 


\section{University Library}

\section{- M M I N E R VA A gateway to Melbourne's research publications}

Minerva Access is the Institutional Repository of The University of Melbourne

Author/s:

Ejima, $\mathrm{H}$;Richardson, JJ;Caruso, F

Title:

Multivalent Directed Assembly of Colloidal Particles

Date:

2013-01-01

Citation:

Ejima, H., Richardson, J. J. \& Caruso, F. (2013). Multivalent Directed Assembly of Colloidal Particles. ANGEWANDTE CHEMIE-INTERNATIONAL EDITION, 52 (12), pp.3314-3316. https://doi.org/10.1002/anie.201209461.

Persistent Link:

http://hdl.handle.net/11343/123304 